\title{
Configuring the cancellation of optical near-fields
}

\author{
David. L. Andrews, Justo Rodríguez, Luciana C. Dávila Romero \\ Nanostructures and Photomolecular Systems, School of Chemical Sciences, \\ University of East Anglia, Norwich NR4 7TJ, U.K.
}

\begin{abstract}
The characteristic near-field behavior of electromagnetic fields is open to a variety of interpretations. In a classical sense the term 'near-field' can be taken to signify a region, sufficiently close to some primary or secondary source, that the onset of retardation features is insignificant; a quantum theoretic explanation might focus more on the large momentum uncertainty that operates at small distances. Together, both near-field and wave-zone (radiative) features are fully accommodated in a retarded resonance propagation tensor, within which each component individually represents one asymptotic limit - alongside a third term that is distinctly operative at distances comparable to the optical wavelength. The propagation tensor takes different forms according to the level of multipole involved in the signal production and detection. In this presentation the nature and symmetry properties of the retarded propagation tensor are explored with reference to various forms of electric interaction, and it is shown how a suitable arrangement of optical beams can lead to the complete cancellation of near-fields. The conditions for such behavior are fully determined and some important optical trapping applications are discussed.
\end{abstract}

Keywords: Near-field optics, optical trapping, optical binding, optical energy landscapes, nanomanipulation

\section{INTRODUCTION}

Attractive interactions between non-polar molecules in their ground states generally result from so-called dispersion interactions. The first descriptions of such molecular interactions, beyond the range of significant overlap between electron distributions, resulted in the well-known London formula [1], in which the dispersion energy displays an $R^{-6}$ dependence on distance separation. The dispersion forces were originally considered to be the result of second order electrostatic interactions. Although valid at short distances, theory cast in these terms could not include any effects associated with the finite speed of electromagnetic propagation. It is now known that a complete description of intermolecular interactions, applicable at all distances, must accommodate such retardation effects. One of the most important consequences of including retardation is manifest in a replacement of the London dispersion energy distancedependence by a Casimir-Polder term, derived using quantum electrodynamics (QED). In the Casimir-Polder interaction, the asymptotic form of distance dependence for large intermolecular separations changes to $R^{-7}$ [2]. The adoption of calculational methods based on QED, ensuring a formulation of intermolecular coupling correctly encompassing retardation effects, also shows that the Coulomb's law form of interaction between transition dipoles is a limit only applicable at short distances, where retardation may be neglected. The more general result involves a retarded resonance propagation tensor, which accommodates both near-field and wave-zone regions - plus a third term which becomes equally significant in a region of distances comparable to the optical wavelength [3].

In this paper we show that, due to the particular features of this retarded propagation tensor, the effects of near-field interaction may be cancelled when a suitable optical geometry is set up. Such a possibility has important consequences in the case of laser optical trapping, where an interplay of the optically induced interactions between particles significantly impinges on the viability, creation and stability of array configurations. Configuring a collapse of the optical binding interactions can completely collapse such structures.

In the following Section 2, theory is first developed in terms of a retarded propagation tensor, shown to take different forms according to the level of multipole involved in signal production and detection. The nature and symmetry properties of the propagation tensor are explored in Section 3, with reference to various forms of multipolar interaction. In Section 4, the concept of optical binding as a coupling between optical centers is introduced and the conditions for

Metamaterials: Fundamentals and Applications, edited by Mikhail A. Noginov, Nikolay I. Zheludev, Allan D. Boardman, Nader Engheta, Proc. of SPIE Vol. 7029, 70290C, (2008) · 0277-786X/08/\$18 · doi: 10.1117/12.796077 
near-field cancellation are thereby determined. In the following section some important optical trapping applications are discussed. Finally, in section 6 we discuss and summarize our results.

\section{QED FORMULATION OF MULTIPOLAR COUPLING}

We begin by considering a simple system comprising two electronically distinct and neutral molecules, $A$ and $B$. In this system, the process to be addressed entails interactions of $A$ and $B$ with light. In the multipolar form of quantum electrodynamics the Hamiltonian for this system may be represented as;

$$
H=H_{\mathrm{rad}}+\sum_{\xi=A, B} H_{\mathrm{at}}(\xi)+\sum_{\xi=A, B} H_{\mathrm{int}}(\xi) .
$$

Here $H_{\mathrm{rad}}$ is the Hamiltonian for the radiation field in vacuo, $H_{\mathrm{at}}(\xi)$ is the field-free Hamiltonian for a molecule $\xi$, and $H_{\text {int }}(\xi)$ represents the Hamiltonian for the interaction of the molecule with the radiation field. The tripartite simplicity of equation (1) results from adoption of the multipolar form of light-matter interaction, derivable from minimal-coupling interaction by a well-known canonical transformation. It can be argued that any multipolar form is, indeed, rigorous only within a quantum electrodynamical formulation [4].

For present purposes the Hamiltonian of interaction between the electromagnetic field with functionally identical particles $(A, B)$ is expressible as follows;

$$
H_{\text {int }}(\xi)=-\int \boldsymbol{p}^{\perp}(\xi, \boldsymbol{r}) \cdot \boldsymbol{e}^{\perp}(\boldsymbol{r}) d^{3} \boldsymbol{r} .
$$

The transverse electric polarization field $\boldsymbol{p}^{\perp}(\xi, \boldsymbol{r})$ is succinctly expressible in a form (3) whose expansion in powers of $\lambda$ delivers the entire multipolar series [5];

$$
\boldsymbol{p}^{\perp}(\xi, \boldsymbol{r})=\sum_{\alpha(\xi)} e_{\alpha}\left(\boldsymbol{q}_{\alpha}-\boldsymbol{R}_{\xi}\right) \int_{0}^{1} \delta^{\perp}\left(\boldsymbol{r}-\boldsymbol{R}_{\xi}-\lambda\left(\boldsymbol{q}_{\alpha}-\boldsymbol{R}_{\xi}\right)\right) d \lambda,
$$

where the summation is taken over the constituents of molecules $\xi$ whose centers of mass are located at $\boldsymbol{R}_{\xi}$, their constituent charges $e_{\alpha}$ located at positions $q_{\alpha}$. In application to any specific process or interaction, it is necessary to consider only the form of coupling effected by the lowest order of electric multipole that supports each associated donor and acceptor transition. A traceless form can be adopted for the quadrupole and higher order multipoles, consistent with the transverse character of the electric displacement field [6]. Using the implied summation convention for repeated tensor and vector indices, the Hamiltonian for the interaction may be re-expressed;

$$
H_{\mathrm{int}}^{\xi}=-\varepsilon_{o}^{-1} \sum_{\xi=\mathrm{A} . \mathrm{B}} \mu_{i}(\xi) d_{i}^{\perp}\left(\mathbf{R}_{\xi}\right)-\varepsilon_{o}^{-1} \sum_{\xi=\mathrm{A} . \mathrm{B}} Q_{i j}(\xi) \cdot \nabla_{j} d_{i}^{\perp}\left(\mathbf{R}_{\xi}\right)-\ldots,
$$

where $\boldsymbol{\mu}(\xi)$ and $\mathbf{Q}(\xi)$ are the corresponding operators for the electric dipole and electric quadrupole. The second term in (4), typically smaller than the first by a factor of the order of the fine structure constant (i.e. two-three orders of magnitude smaller) takes the lead in a series of higher order multipole corrections (for the later calculations, only the leading term is retained). The electric displacement vector field $\mathbf{d}^{\perp}$ in (4) is itself expressible, using the Power-ZienauWoolley approach, as a mode expansion that is linear in the photon creation and annihilation operators [7].

For the coupling between two electric multipoles $\mathrm{E} m$ - $\mathrm{E} n$ (where $\mathrm{E} n$ is used to denote an electric multipolar interaction of order $n$ - for example E2 denotes the electric quadrupole), quantum electrodynamical calculation delivers a result that can be cast as follows [8-10];

$$
M_{f i}^{\mathrm{E} m-\mathrm{E} n}=E_{A ; i_{1} \ldots i_{m}}^{(m)} V_{i_{1} \ldots i_{m} j_{1} \ldots j_{n}}(k, \mathbf{R}) E_{B ; j_{1} \ldots j_{n}}^{(n)},
$$


where $\boldsymbol{E}_{\xi}^{(m)}$ is the transition multipole tensor of order $m$ for component $\xi$, and the coupling tensor, quantifying the propensity for an $\mathrm{E} m$ transition at $A$ to be electromagnetically coupled with an $\mathrm{E} n$ transition at $B$, is expressed as follows;

$$
V_{i_{1} \ldots i_{m} j_{1} \ldots j_{n}}(k, \mathbf{R})=\frac{(-1)^{m}}{4 \pi \varepsilon_{0}} \nabla_{i_{2}} \ldots \nabla_{i_{m}} \nabla_{j_{2}} \ldots \nabla_{j_{n}}\left(-\nabla^{2} \delta_{i_{1} j_{1}}+\nabla_{i_{1}} \nabla_{j_{1}}\right) \frac{e^{i k R}}{R}
$$

also $k=\Delta E / \hbar c$, where $\Delta E$ is the energy exchanged in the interaction; $\mathbf{R}$ is the displacement vector defined by $\mathbf{R}_{\mathrm{B}}-\mathbf{R}_{\mathrm{A}}$. It also emerges from the analysis that precisely the same formula, (6), operates for the coupling between magnetic multipoles [8]. The rank $(m+n)$ coupling tensor $V_{i_{1} \ldots i_{m} j_{1} \ldots j_{n}}(k, \boldsymbol{R})$, given in equation (6), is also called the retarded potential tensor. Its retarded characteristic applies over all distances, accommodating readily identifiable short-range (near-field, $k R \square 1$ ) and long-range (wave-zone, $k R \square 1$ ) asymptotes. The former behavior is identified with virtual photon coupling (resonance energy transfer) - the latter, real photon propagation (radiative transfer) [11]. Thus, as the detector is moved outwards, away from the near-field, a progressively radiative character to the energy transfer emerges. From a quantum theoretic viewpoint, this reflects the fact that as the distance between the coupled multipoles increases, there is a decreasing uncertainty in the co-aligned electromagnetic momentum [3].

The simplest and most significant form of electric multipole coupling is the well-known solution for dipole-dipole interaction [12];

$$
M_{f i}^{\mathrm{E} 1-\mathrm{E} 1}=\mu_{A i} V_{i j}(k, \mathbf{R}) \mu_{\mathrm{B} j},
$$

where;

$$
V_{i j}(k, \mathbf{R})=\frac{e^{i k R}}{4 \pi \varepsilon_{0} R^{3}}\left\{(1-\mathrm{i} k R)\left(\delta_{i j}-3 \hat{R}_{i} \hat{R}_{j}\right)-(k R)^{2}\left(\delta_{i j}-\hat{R}_{i} \hat{R}_{j}\right)\right\}
$$

is the lowest rank tensor given by equation (6). The calculation that leads to (8) involves a summation over modes for a virtual photon propagating in either direction between $A$ and $B$. From this result, it may be interpreted that, consistent with the E1 nature of the coupled transitions, the mediating virtual photon conveys one unit of angular momentum irrespective of the detector displacement form the source. This, however, is an interpretation whose extension to higher multipoles has a potential pitfall. For example, it is not widely understood that electric quadrupole decay produces radiation that is detectable not only by quadrupole detectors, but also by a dipole detector - yet a clearly non zero result [9,11-13] emerges for the latter case of E2-E1 coupling. This and other processes are both fully allowed and addressed by the general QED theory; in all cases the source-detector coupling is achieved by regular spin-1 photon [14].

\section{SYMMETRY PROPERTIES OF THE COUPLING TENSOR}

To progress further, an irreducible tensor construction of the three tensors in expression (5) may be introduced. Though spherical tensors are commonly engaged for the analysis of multipole transitions [15], an irreducible Cartesian tensor formulation [16] facilitates a more direct connection with the development given in the previous Section, and it conveys the same physical information. The connection between the two representations is well established [17]. The first step is decomposition of the three tensor forms in equation (6) into irreducible parts. In general, a Cartesian $n$-rank tensor comprises irreducible constituents of integer weights $j$ in the interval $(0 \leq j \leq n)$, each $j$ also signifying the associated angular momentum. The decomposition is trivial in the case of the multipole moments; since they are fully indexsymmetric and traceless, each is properly and uniquely expressible as a natural tensor, i.e. one whose weight equals its rank. This is a reflection of the local spherical symmetry that applies to an atom source or detector. It is not, however, a feature of any of the coupling tensors $\mathbf{V}(k, \mathbf{R})$ delivered by equation (5); the entirety of the source-detector system is clearly of less than spherical symmetry. For example, the E1-E1 result (7) comprises weights 0 and 2; a prescription for their determination is readily available [18]. It can be easily seen that in the near-zone $(k R \square 1)$, the term with the highest inverse power of $R$ dominates $V_{i_{1} \ldots i_{m} j_{1} \ldots j_{n}}(k, \boldsymbol{R})$, and that term entails weight $(m+n)$ alone. Hence, in short range, any coupling that leads to the evaluation of a weight 0 part of the $V_{i_{1} \ldots i_{m} j_{1} \ldots j_{n}}(k, \mathbf{R})$ tensor, such as arises in the case of 
isotropic systems, will result in a zero contribution. A more explicit description of the coupling tensor in terms of its irreducible parts can be found in reference [14]. In the following section we consider a specific case of coupling.

\section{OPTICAL BINDING AND ITS CANCELLATION}

A well-known example of optically-induced coupling between two optical centers is afforded by optical binding [19], where the optical coupling of particles $A$ and $B$ is induced by throughput, moderately intense, laser radiation.. A suitable basis set for calculating the potential energies and forces associated with such processes comprises products of the electronic states of each particle, and a number state for each mode of the radiation field; the particle pair energies emerge as diagonal elements of the perturbation matrix. The optically induced coupling can be described by four entangled E1 interactions, the annihilation and creation events of one real and one virtual photon. Since each operation of the electromagnetic field entails a photon creation or annihilation, the leading term in the system energy is delivered by fourth-order perturbation theory. Casting the result in terms of physically measurable quantities, and using the explicit result (8) for E1-E1 coupling, the following result emerges for the optically induced shift in the inter-particle potential energy, produced by a beam of irradiance $I[20]$;

$$
\Delta E_{i n d}(\mathbf{k}, \mathbf{R})=\left(\frac{I}{\varepsilon_{0} c}\right) \operatorname{Re}\left[e_{i}^{(\lambda)} \bar{e}_{l}^{(\lambda)} \alpha_{i j}^{\mathrm{A}}(k) V_{j k}(k, \mathbf{R}) \alpha_{k l}^{\mathrm{B}}(k)\right] \cos (\mathbf{k} \cdot \mathbf{R}),
$$

where $e_{i}^{(\lambda)}$ is the $i$ th component of the laser polarization vector, $\alpha_{i j}^{\xi}(k) \equiv \alpha_{i j}^{\xi}(-k, k)$ is the frequency-dependent dynamic polarizability of a particle $\xi$ [18], and $V_{j k}(k, \mathbf{R})$ is the fully retarded resonance dipole-dipole interaction tensor. No other contributions arise at this order $\left(E 1^{4}\right)$ if the particles are centrosymmetric and non-polar, as will be assumed in the following. (In cases where $A$ and $B$ are polar, other contributions will arise, signifying real photon annihilation and creation events, co-located at one or the other particle).

It can be shown that the near-field effects of optical binding may be cancelled out for particular configurations. The principle of such assertion can be easily shown. Let us consider the case where there is throughput radiation derived from multiple beams, with their polarizations disposed in all three directions, Fig. 1. It can be readily seen that the associated energy is:

$$
\Delta E_{\text {ind }}^{3 \text { beams }}(\mathbf{k}, \mathbf{R})=\left(\frac{I \alpha_{o}^{\mathrm{A}}(k) \alpha_{0}^{\mathrm{B}}(k)}{\varepsilon_{0} c}\right) \operatorname{Re}\left[V_{x x}(k, \mathbf{R})+V_{y y}(k, \mathbf{R})+V_{z z}(k, \mathbf{R})\right] \cos (\mathbf{k} \cdot \mathbf{R})
$$

In the near-field zone, we have the phase factor $\cos (\mathbf{k} \cdot \mathbf{R}) \rightarrow 1$ and the retarded potential contribution, $V_{x x}^{N F}(k, \mathbf{R})+V_{y y}^{N F}(k, \mathbf{R})+V_{z z}^{N F}(k, \mathbf{R})=0$, such that the right-hand side of (10) vanishes; the short-range optically induced fields completely cancel.

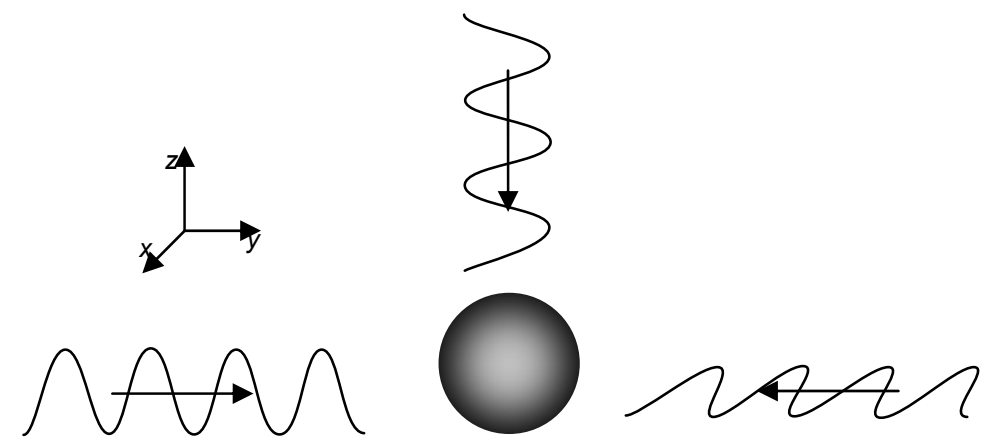

Fig. 1. Scheme showing the geometry of an isotropic optical input, ignoring radiative pressure. Each beam has linear polarization in one of three axes. In the near-zone region, the inter-particle separation $\mathbf{R}$ is smaller that the wavelength $\lambda$. 
It is important to note that expression (10) does not account for the effects of radiative pressure usually present in optical binding process. To counter the latter effects, experimental observations (such as on cold and ultracold molecules, optically trapped nanoparticles, microparticles and colloids) are usually entertained in counter-propagating laser beams. This scheme ensures that beam radiation pressure cancels out, and any optically induced potential is the only significant force over nanoscale distances where the irradiance is effectively constant [21]. A modification of the latter laser set-up is shown in the following Section to cancel optical near-fields.

\section{OPTICAL TRAPPING APPLICATION}

Here, we consider one set-up of counter-propagating laser beams, supplemented by the addition of a secondary laser throughput designed to modify the total field the particles experience. With reference to a simple particle pair, Fig. 3 illustrates the wave-vector and polarization vector geometry, also defining parameters for establishing their orientation relative to the inter-particle displacement vector. Two counter-propagating beams (primary beams $\mathbf{k}_{1}$ and $\mathbf{k}_{2}$, the standard configuration) are located on the $x$-axes, each having the same polarization in the $y$ direction and with an optical field of magnitude $E$ (or irradiance $I$ ). These beams are intersected orthogonally by a secondary field, $\mathbf{k}_{3}$, propagating in the y direction with $x$-axis polarization; the latter, on mirror reflection, propagates back as $\mathbf{k}_{4}$ with a polarization rotated by $\pi / 2$ (through the employment of an optical element not shown in the diagram). The field amplitude of the secondary beam and its reflection are $\sqrt{2} E$, which is equivalent to an irradiance of $2 I$. Previous studies has shown that modifying the irradiance of the secondary field in this setting, multiple particles in the near-field may be continuously transformed between linear, spherical and lamellar forms, and optically trapped and bound micron-sized chain of particles may collapse [22]. Here, this laser setting is shown to cancel the optical near-fields that are characterized by an inverse third power dependence on particle separation.

At the intersection of the fields of the four laser beams as described, it can be shown from equation (9) that particles experience pair potentials given by;

$$
\Delta E=\left(\frac{I k^{3} \alpha_{0}^{A} \alpha_{0}^{B}}{16 \pi^{2} c \varepsilon_{0}^{2}}\right)\left(\left[C_{1}+D_{1}\right] \frac{\cos k R}{k R}+\left[C_{3}+D_{3}\right]\left(\frac{\cos k R}{k^{3} R^{3}}+\frac{\sin k R}{k^{2} R^{2}}\right)\right),
$$

where $C_{n}=\left(1-n \cos ^{2} \phi\right) \cos (k R \sin \phi \cos \zeta)$ and $D_{n}=\left(2-n \sin ^{2} \phi\right) \cos (k R \cos \phi)$. Developing equation (11) for the short range $k R<<1$, by the use of l'Hôpital's rule and the near-field approximations $\sin (k R) \cong k R$ and $\cos (k R) \cong 1$, the interparticle potential may be expressed as;

$$
\Delta E=-\left(\frac{k^{3} \alpha_{0}^{A} \alpha_{0}^{B}}{16 \pi^{2} c \varepsilon_{0}^{2}}\right)\left[\frac{2}{k R}+\frac{\left(2-3 \sin ^{2} \phi\right)\left(\cos ^{2} \phi-\sin ^{2} \phi \cos ^{2} \zeta\right)}{3 k R}\right] .
$$

In this way, the inverse power dependent term of the dipole-dipole coupling, which only dominates for particles separated by distances of the order of the optical wavelength, becomes the sole surviving term in the near field zone.

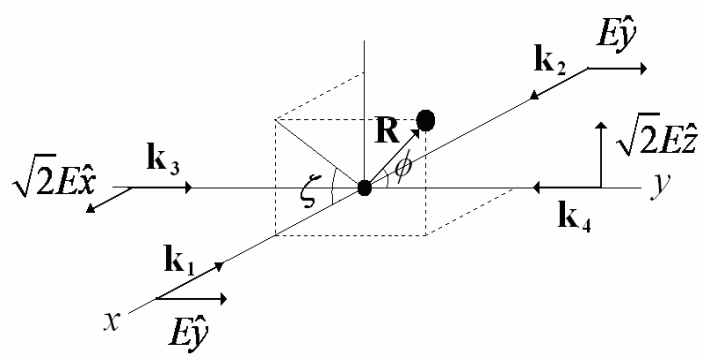

Fig. 3. Pair of particles irradiated by two counter-propagating beams, $\left(E \hat{\mathbf{y}}, \mathbf{k}_{1}\right)$ and $\left(E \hat{\mathbf{y}}, \mathbf{k}_{2}\right),($ standard optical binding) and beam $\left(\sqrt{2} E \hat{\mathbf{x}}, \mathbf{k}_{3}\right)$ reflected with transverse polarization as $\left(\sqrt{2} E \hat{\mathbf{z}}, \mathbf{k}_{4}\right)$. The angle subtended by the inter-particle displacement vector $\mathbf{R}$ on the $y$-axis is $\phi$, and $\zeta$ is the angle between the $\mathbf{R}$ projection on the $z x$-plane and the $x$-axis. 
The behavior that can be expected, when particles initially trapped in a conventional counter-propagating beam setup are subjected to secondary beams of increasing intensity, can now be discussed. At first, with particles separated by small distances, the Casmir-Polder potential might be considered to be of considerable importance due to its inverse sixth power distance dependence, enabling a group of trapped particles to form a compact structure. However, [23] the laser irradiances employed in optical trapping are usually high enough for optical binding forces to dominate, and the particles line up along the polarization direction. By smoothly increasing the irradiance of the secondary laser $\mathbf{E}_{3}$ and $\mathbf{E}_{4}$ (and consequently also the reflected field), the particle system will be passively modified to a spherical form when the irradiance of the secondary field becomes equal to the irradiance of the counter-propagating beams. On any further increase of the secondary beam irradiance, the sphere will be continuously modified to a lamellar form, transverse to the secondary field propagation direction. The initial structure is then recoverable by reducing the irradiance of the secondary beam. In summary, within the near-field zone, the optical binding forces and the structures that they create can be modified and even cancelled for particular arrangements and relative intensities of the optical beams involved.

\section{CONCLUSION}

The nature and symmetry properties of the electric multipolar coupling tensor have been briefly explored with reference to various forms of interaction. It has been shown that optical binding effects can be cancelled in the near-field zone when the particles are subjected to multidirectional optical radiation. In particular, by introducing a secondary pair of counter-propagating laser beams, the observation of optical binding has been shown to cancel the near-fields that are involve in the interparticle interaction; thus the pair energy has an inverse power dependence in the near-zone which is attractive in nature.

\section{ACKNOWLEDGEMENT}

This work is in part funded by the Engineering and Physical Sciences Research Council.

\section{REFERENCES}

[1] London, F. ,"Zur Theorie und Systematik der Molekularkräfte”, Zeitschrift für Physik, 63, 245-279 (1930)

[2] Casimir, H. B. G. and Polder, D., "The Influence of Retardation on the London-van der Waals Forces" Physical Review,. 73, 360-372 (1948).

[3] Andrews, D.L. and Bradshaw, D.S. "Virtual photons, dipole fields and energy transfer: a quantum electrodynamical approach,” Eur. J. Phys. 25, 845-858 (2004).

[4] R. G. Woolley, "Gauge invariance and multipole moments", Adv. Quantum Chem., 32, 167-180 (1999)

[5] Babiker, M. , Power, E. A., and Thirunamachandran, T., "Generalization of Power-Zienau-Woolley transformation in quantum electrodynamics and atomic field equations", Proc. Roy. Soc. Lond. A: Math. Phys. Eng. Sci., 338, 235249 (1974)

[6] Gray, C. G., and Gubbins, K. E., [Theory of Molecular Fluids], University Press, Oxford, 51-53, (1984).

[7] Craig, D. P., and Thirunamachandran, T., [Molecular Quantum Electrodynamics], Dover, Mineola, New York, (1998).

[8] Scholes, G. D. and Andrews, D. L., "Damping and higher multipole effects in the quantum electrodynamical model for electronic energy transfer in the condensed phase", J. Chem. Phys,. 107, 5374-5384 (1997)

[9] Salam. A., "A general formula for the rate of resonant transfer of energy between two electric multipole moments of arbitrary order using molecular quantum electrodynamics", J. Chem. Phys., 122, 044112 (2005).

[10] Salam, A., "Intermolecular interactions in a radiation field via the method of induced moments," Phys. Rev. A. 73, 013406 (2006).

[11] Jenkins, R. D., Daniels, G. J. and Andrews, D. L. "Quantum pathways for resonance energy transfer," J. Chem. Phys, 120, 11442 (2004).

[12] G. Juzeliūnas and D.L. Andrews, "Quantum electrodynamics of resonance energy transfer," Adv. Chem. Phys., 112, $357-410(2000)$ 
[13] Bradshaw, D. S., "Quantum electrodynamical analysis of nonlinear optical effects deriving from the laser irradiation of molecular systems: Resonance energy transfer and optomechanical forces", $\mathrm{PhD}$ thesis (University of East Anglia, Norwich, UK, 2005).

[14] Andrews, D. L., "Optical angular momentum: Multipole transitions and photonics" submitted for publication (2008)

[15] Grinter, R., "Photon angular momentum: selection rules and multipolar transition moments" J. Phys. B: At. Mol. Opt. Phys. 41, 095001 (2008)

[16] Coope, J. A. R, Snider, R. F., and McCourt, F. R., "Irreducible Cartesian Tensors", J. Chem. Phys. 43, 2269-2275 (1965).

[17] Jerphagnon, J., Chemla, D., and Bonneville, R., "The description of the physical properties of condensed matter using irreducible tensors", Adv. Phys. 27, 609-650 (1978).

[18] Andrews, D. L., and Allcock, P.,[Optical Harmonics in Molecular Systems], Wiley-VCH, New York (2002).

[19] Dávila Romero, L. C. and Andrews, D. L., [Structured Light and Its Applications: An Introduction to PhaseStructured Beams and Nanoscale Optical Forces], ed. D. L. Andrews, Academic, Burlington MA,, 79-105. (2008)

[20] Bradshaw, D. S., and Andrews,. D. L., "Optically induced forces and torques: Interactions between nanoparticles in a laser beam", Phys. Rev. A. 72, 033816 (2005).

[21] Metzger, N. K., Marchington, R. F., Mazilu, M., Smith, R. L., Dholakia, K. and Wright, E. M., "Measurement of the restoring forces acting on two optically bound particles from normal mode correlations," Phys. Rev. Lett. 98, 068102 (2007).

[22] Andrews, D. L. and Rodríguez, J. "Collapse of optical binding under secondary irradiation" Opt Lett., to be published (2008)

[23] Rodríguez, J., Dávila Romero, L. C. and Andrews, D. L,. “Optically induced potential energy landscapes,” J. Nanophotonics. 1, 019503 (2007). 